\title{
Adaptação Transcultural e Validação das Propriedades Psicométricas do Instrumento Nutrition in Patient Care Survey (Nips) no Brasil
}

\section{Cross Cultural Adaptation and Validation of Psychometric Properties of the Instrument Nutrition in Patient Care Survey (Nips) in Brazil}

\author{
Juliana Peres Sleumer \\ Ângelo Ponte de Freitas Campos ${ }^{I}$ \\ Felipe Anastácio da Silva Machado \\ Eliane Perlatto Moura ${ }^{I \mathbb{C}}$
}

\section{PALAVRAS-CHAVE}

- Questionário.

- Ciências da Nutrição.

- Atitudes e Prática em Saúde.

- Educação Médica.
Introdução: O hábito nutricional inadequado é considerado importante fator de risco para o desenvolvimento de doenças crônicas não transmissíveis (DCNT). O médico tem importante papel na modificação e melhoria do comportamento nutricional de seus pacientes e consequente melhoria da saúde. Neste contexto, é necessário mensurar, mediante instrumentos confiáveis, a atitude de estudantes e médicos sobre nutrição no atendimento a pacientes, para subsidiar intervenções educacionais que impactarão a prática clínica. Objetivo: Traduzir, adaptar culturalmente e validar o instrumento Nutrition in Patient Care Survey (Nips), que avalia atitudes no cuidado nutricional na prática clínica, para ser utilizado na população de língua portuguesa falada no Brasil. Metodologia: A versão original Nips em inglês inicialmente foi traduzida para a lingua portuguesa e submetida a uma adaptação transcultural do vocabulário e da construção linguística. Esta versão foi retraduzida para o inglês. $O$ questionário foi então aplicado para pré-teste em 30 estudantes de Medicina, com o intuito de eliminar dúvidas quanto à compreensão das perguntas. A versão final do instrumento adaptado foi aplicada a 400 estudantes de Medicina. Foi realizada a análise de confiabilidade e de validade, utilizando-se análise fatorial exploratória pelo método de extração de componentes principais e rotação Varimax Resultado: Quatrocentos estudantes responderam a todas as perguntas do Nips e foram incluídos na análise. Após análise fatorial, foi definida a estrutura final da escala, que passou a ter 37 itens, em formato Likert de 1 (discordo totalmente) a 5 (concordo totalmente), divididos em dez fatores, diferentemente do observado na escala original, que apresenta cinco fatores. A consistência interna (alfa de Cronbach) para os dez fatores estudados foi superior a 0,60 na maioria deles, e o alfa de Cronbach geral foi superior a 0,50. Na análise de validade do construto, a maioria dos coeficientes de correlação se mostrou significativa (valores $p<0,005$ ). Com essa estrutura, a escala proposta apresentou consistência interna entendida como favorável, explicando 58,93\% das atitudes dos alunos em relação ao cuidado nutricional na assistência ao paciente. Conclusão: A versão em português do instrumento Nips, após o processo de adaptação, mostrou-se útil e adequada para levantar informações relativas à atitude dos estudantes de Medicina no cuidado nutricional do paciente. 


\section{KEY-WORDS}

- Questionnaire.

- Nutritional Sciences.

- Attitudes.

- Practice.

- Medical education.

Recebido em: 17/3/19

Aceito em: 1/4/19

\section{INTRODUÇÃO}

Na América Latina, a prevalência de obesidade é a maior do mundo, alcançando $62 \%$ na população adulta com idade superior a 20 anos. No Brasil, metade da população adulta e um terço das crianças (a partir de cinco anos) e adolescentes são considerados obesos ${ }^{1}$. Neste contexto, a educação nutricional da população torna-se fundamental e requer a atuação de profissionais de saúde que saibam identificar e abordar problemas relacionados à alimentação e nutrição ${ }^{2,3}$.

Vários estudos têm demonstrado que, em muitos casos, as intervenções nutricionais atuam de modo similar ou até mesmo mais efetivo do que as intervenções farmacológicas, além de diminuírem os riscos de complicações em várias enfermidades, bem como o custo da assistência à saúde ${ }^{4,5,6}$.

Observa-se atualmente um movimento global no sentido de mudar os paradigmas vigentes na educação médica. Eventos mundiais sinalizam políticas para ações que garantam uma formação voltada à promoção da saúde integral do ser huma-
ABSTRACT

Introduction: Inadequate nutritional habits are considered a leading risk factor for the development In this context, it is necessary to measure, through reliable instruments, the attitude of students and physicians toward nutrition in patient care, to subsidize educational interventions that will impact initially translated into Portuguese and submitted to a cross-cultural adaptation of vocabulary and linguistic construction. This version was then retranslated into English. The questionnaire was then to pre-test in 30 medical students, in order to eliminate doubts about comprehension of the through principal component extraction and Varimax rotation methods. Result: Four hundred students answered all NIPS questions and were included in the analysis. Following factorial analysis, inal structure of the scale was defined, which now has 37 items, in Likert form ranging from 1 (totally agree), divided into ten factors, which differs from the original scale with 0.60 in most cases and the general alpha was greater than 0.50. In the construct validity analysis, the majority of the correlation coefficients were significant ( $p$ values $<0.005$ ). With this structure, the proposed scale presented an internal consistency understood as favorable, explaining 58.93\% of students' attitudes regarding nutritional care in patient care. Conclusion: The version in Portuguese of the NIPS instrument, after an adaptation process, proved to be useful and adequate for gathering information regarding the attitude of medical students related to nutritional care of patients. 
de nutrição e do aconselhamento nutricional na prática profissional. E ainda, naqueles que consideram o aconselhamento nutricional uma prioridade, percebe-se ausência de confiança e conhecimento para fornecer efetivamente uma educação nutricional adequada aos pacientes ${ }^{9-12}$. Existem poucos relatos na literatura sobre intervenções curriculares que de fato tenham impactado positivamente as atitudes dos estudantes em relação ao aconselhamento nutricional no atendimento clínico. As razões fundamentais para esta ausência de progresso são as práticas educacionais, bem como os instrumentos existentes para medir a atitude dos alunos em relação à nutrição, os quais são descritos como metodologicamente fracos. É necessário mensurar por meio de instrumentos confiáveis a atitude de estudantes e médicos em relação à nutrição no atendimento a pacientes para subsidiar intervenções educacionais que impactarão a prática clínica ${ }^{13}$.

Diante disto e devido à ausência de instrumentos validados que abordem o tema em questão na língua portuguesa, o objetivo deste estudo foi realizar a adaptação transcultural do Nips, desenvolvido por McGaghie et al. ${ }^{13}$, para o português falado no Brasil e validar as propriedades psicométricas da escala por meio da análise fatorial com o intuito de disponibilizar esta ferramenta para estudos de atitude de estudantes de Medicina em relação ao cuidado nutricional do paciente no Brasil.

\section{MÉTODOS}

\section{Desenho do estudo}

Trata-se de um estudo transversal de metodologia quantitativa, cuja proposta foi adaptar e validar um instrumento para mensurar a atitude dos estudantes de Medicina em relação ao aconselhamento nutricional, no atendimento clínico. Este estudo foi dividido em duas fases: Fase 1 - tradução e adaptação transcultural; Fase 2 - avaliação das propriedades psicométricas do instrumento traduzido.

\section{Instrumento}

Em revisão bibliográfica realizada nas bases de dados Pubmed, Medline e SciELO, não foi encontrado um instrumento capaz de medir as atitudes dos estudantes de Medicina validado na língua portuguesa do Brasil. Diante disto, optou-se por validar o instrumento Nutrition in Patient Care Survey (Nips), elaborado por McGaghie et al. ${ }^{13}$, com a permissão dos autores. A escolha do Nips $^{13}$ baseou-se no fato de se tratar de um instrumento sistematicamente elaborado e validado para mensurar atitudes de estudantes de Medicina e residentes em relação à nutrição na assistência ao paciente e que é referenciado em vários estudos sobre o tema ${ }^{12,14,15}$.
O Nips é composto por 45 questões, agrupadas em cinco domínios: 1 - Nutrição no atendimento de rotina (8 itens); 2 - Comportamento clínico (20 itens); 3 - Relacionamento médico-paciente (8 itens); 4 - Comportamento do paciente / nutrição (3 itens); 5 - Eficácia médica (6 itens). Trata-se de um questionário com 25 questões com resposta numa escala do tipo Likert com cinco pontos e 20 questões com respostas dicotômicas do tipo sim/não ${ }^{13}$. O escore total do instrumento varia de 45 a 145, sendo que, quanto maior o escore, melhor a atitude dos estudantes de Medicina em relação ao aconselhamento nutricional na prática clínica. Segundo os autores, cada subescala produz dados confiáveis em termos de consistência interna (coeficiente alfa de Cronbach) e estabilidade (confiabilidade teste-reteste).

Conforme realizado por Crowley et al. ${ }^{15}$ e Walsh et al. ${ }^{14}$, optou-se, também, neste estudo, por utilizar a escala de Likert de 1 a 5 (1 - discordo totalmente; 2 - discordo; 3 - não concordo nem discordo; 4 - concordo; 5 - concordo totalmente), pois ela permite maior amplitude de respostas ${ }^{16}$.

\section{Fase 1 - Tradução e adaptação cultural}

Tradução: O processo de tradução e adaptação cultural seguiu os passos sugeridos por Beaton et al. ${ }^{17}$. Essa etapa consistiu na tradução da versão original do instrumento em inglês para a língua portuguesa, realizada por dois profissionais com inglês fluente, sendo um profissional da área médica/nutrição e outro da área não médica, com formação de nível superior e proficiência na língua inglesa, de modo que fossem consideradas não só as peculiaridades do tema estudado, como a linguagem usada pela população em geral.

Síntese: As duas traduções foram comparadas, e as divergências foram anotadas e discutidas pelos pesquisadores, obtendo-se, então, após consenso, uma versão única.

Retrotradução: Com base na síntese do questionário, a última versão foi convertida para o inglês por dois retrotradutores, que eram cidadãos ingleses que vivem no Brasil, fluentes nas duas línguas. Esta etapa não apresentou discrepâncias relevantes e mostrou equivalência semântica com o instrumento original, assegurando a consistência da tradução.

Revisão pelos autores: O objetivo desta etapa foi produzir a versão final do instrumento. Instruções, itens, formato do instrumento, escala de respostas, entre outras questões, foram discutidos entre os pesquisadores.

Adaptação semântica e cultural (pré-teste): Segundo Beaton et al. ${ }^{17}$, o instrumento deve ser aplicado entre 30 a 40 indivíduos numa amostra da população-alvo, com a finalidade de verificar as dúvidas e a compreensão dos itens do instrumento. A adaptação semântica e cultural da versão traduzida 
foi, então, realizada em um estudo piloto feito com 30 estudantes de Medicina da Universidade José do Rosário Vellano (Unifenas-BH), selecionados aleatoriamente, sendo 18 do sexo feminino e 12 do sexo masculino, com idades variando entre 19 e 34 anos, distribuídos da seguinte forma: 23 alunos de 19 a 23 anos, 3 alunos de 25 a 30 anos, e 4 alunos com mais de 30 anos. Nesta etapa foi avaliada a compreensão dos estudantes em relação a cada item do instrumento (Figura 1).

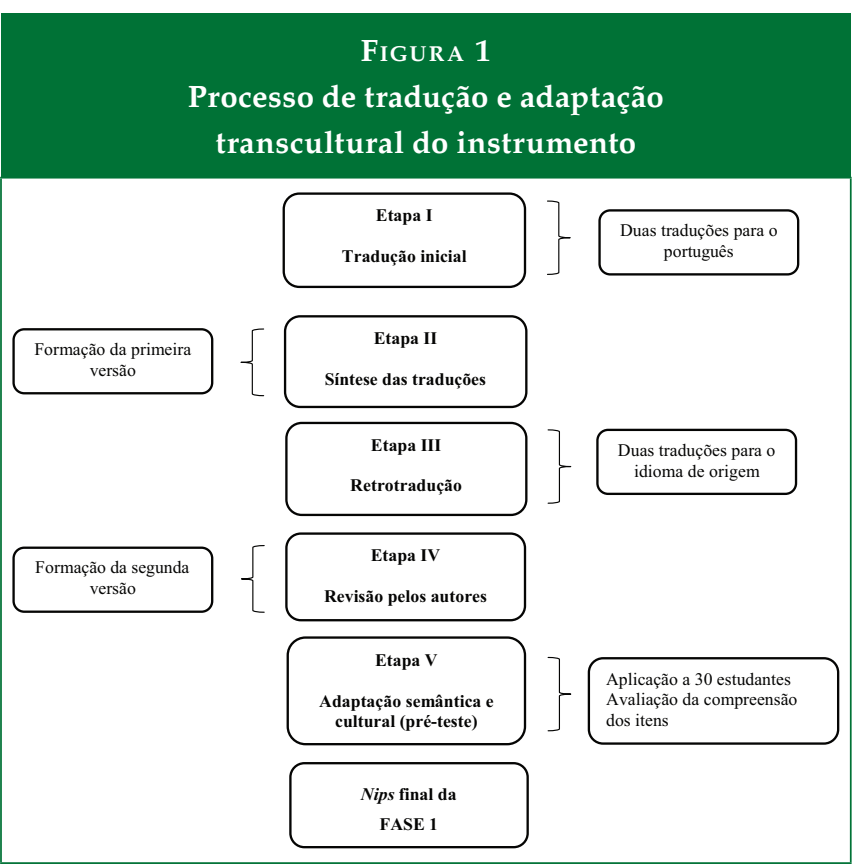

Fonte: Adaptado de Beaton et al. ${ }^{17}$.

Após o processo de tradução e adaptação cultural e aceite do Comitê de Ética em Pesquisa - Coep/Unifenas com o número CAAE 60247416.7.0000.5143, iniciou-se a coleta dos dados.

\section{Fase 2 - Avaliação das propriedades psicométricas do} instrumento

\section{Coleta de dados}

Para a avaliação das propriedades psicométricas do instrumento, este foi distribuído para 400 estudantes de Medicina da Unifenas-BH no período compreendido entre março e abril de 2017. Forneceu-se a cada participante o Termo de Consentimento Livre e Esclarecido, explicando-se os objetivos da pesquisa e aspectos éticos. Após a assinatura do termo, foi aplicado o instrumento de forma coletiva e em caráter voluntário, procurando-se utilizar aulas que apresentassem maior frequência dos estudantes, respeitando-se a disponibilidade do professor para ceder tempo de sua aula. Foram convidados todos os alunos do primeiro ao décimo segundo períodos do curso de Medicina da Universidade José do Rosário Vellano. Buscou-se uma seleção ampla de alunos, do primeiro ao último ano, que representassem os diversos momentos do curso. Os dados foram coletados por meio do preenchimento de questionário autorrespondido, contendo 57 questões, composto por 12 questões sociodemográficas e pela versão traduzida e adaptada do instrumento Nips, resultante da Fase 1 (45 itens). As questões sociodemográficas foram coletadas com a finalidade de caracterizar a amostra, abordando as seguintes variáveis: sexo, idade, período do curso, especialidade pretendida, se possui outra graduação, frequência de refeições saudáveis, tempo destinado às refeições, preocupação com a alimentação, se fez acompanhamento nutricional, se segue alguma linha de alimentação.

\section{Caracterização da amostra}

A amostra foi composta por 400 estudantes de Medicina, sendo que $31,9 \%$ estavam no primeiro e segundo períodos, $26,8 \%$ no terceiro e quarto períodos, $23 \%$ no quinto e sexto períodos, e os demais $(18,3 \%)$ cursavam do sétimo ao décimo segundo período. A amostra se caracterizou por ter predominância do sexo feminino $(59,2 \%)$ e idade média de 22,3 anos. Apenas $5,5 \%$ dos alunos possuíam outra graduação. Entre as especialidades médicas pretendidas, as mais citadas foram Pediatria (13\%), Cardiologia (11,3\%), Ginecologia (9,8\%) e Cirurgia Geral (9,5\%). Em relação aos hábitos alimentares dos alunos, 49\% fazem refeições saudáveis diariamente e $41,5 \%$ de duas a cinco vezes por semana. A maioria dos alunos (56,2\%) destina de 15 a 20 minutos as suas refeições. Preocupam-se com a alimentação 91,3\% dos alunos, 54,3\% já fizeram acompanhamento nutricional com um profissional e a maioria $(96,2 \%)$ não se define segundo alguma linha de alimentação.

\section{Análise fatorial}

Com o objetivo de verificar a validade do construto estudado, foi realizada uma análise fatorial exploratória, utilizando-se o método de extração dos componentes principais e rotação Varimax, sendo que as questões com sentido inverso ao conjunto predominante das questões avaliadas tiveram suas pontuações invertidas. Para avaliar a existência de conveniência do modelo da análise fatorial, foram utilizados o teste de esfericidade de Bartlett e a medida de adequacidade da amostra de Kaiser-Meyer-Olkin (KMO). Valores superiores a 0,5 no teste $\mathrm{KMO}$ indicam que a análise fatorial é adequada para o conjunto de variáveis estudadas. Para verificar se as questões contribuíam de forma significativa na modelagem da análise fatorial, foram utilizadas as medidas de comuna- 
lidade e as medidas de adequacidade da amostra (MSA) para cada uma das questões participantes da análise fatorial final, considerando-se adequados valores para comunalidades e de adequacidade da amostra (MSA) superiores a 0,5019. Avaliou-se a adequacidade, os autovalores e o percentual de variância explicada, para analisar a conveniência e qualidade do modelo estudado.

O coeficiente alfa de Cronbach foi utilizado para avaliar a confiabilidade da consistência interna de cada um dos fatores determinados pela análise fatorial. Este coeficiente varia de 0 a 1 , sendo que um valor abaixo de 0,5 é certamente tido como baixo; valores acima de 0,6 são geralmente considerados aceitáveis; acima de 0,7, bons; e acima de 0,8, ótimos ${ }^{18}$. A determinação da composição de cada um dos fatores foi baseada nas cargas fatoriais. Neste estudo, com base no tamanho da amostra, no nível de significância (5\%) e no poder de $80 \%$, considerou-se como carga fatorial significante um valor de referência, em módulo, superior a $0,35^{19}$. Com o objetivo de avaliar se os fatores latentes apontados pela análise fatorial eram independentes, foi realizada uma análise de Correlação de Pearson. Neste caso, correlações nulas ou fracas confirmam uma independência entre os fatores (Tabela 1). Foi utilizado o software estatístico SPSS versão 17 para a realização das análises.

\section{TABELA 1}

Avaliação dos parâmetros da análise fatorial para 0

modelo final do questionário Nips validado no Brasil

\begin{tabular}{|lcc|}
\multicolumn{1}{c}{ Parâmetros de adequacidade } & $\begin{array}{c}\text { Valores } \\
\text { ideais }\end{array}$ & $\begin{array}{c}\text { Valores } \\
\text { observados } \\
\text { no estudo }\end{array}$ \\
\hline KMO (MSA total) & $\geq 0,60$ & 0,854 \\
\hline Teste de esfericidade de Bartlett (p) & $<0,05$ & $<0,0001$ \\
\hline MSA (questões individuais) & $\geq 0,50$ & 0,601 a 0,941 \\
\hline Comunalidade & $\geq 0,50$ & 0,482 a 0,789 \\
\hline Alfa de Cronbach - fatores & $\geq 0,50$ & 0,24 a 0,78 \\
\hline Autovalores dos fatores & $>1,00$ & 1,39 a 10,41 \\
\hline $\begin{array}{l}\text { Total da variância explicada - oito } \\
\text { fatores (\%) }\end{array}$ & $\geq 50 \%$ & $58,93 \%$ \\
\hline Carga fatorial das questões & $>0,35$ & 0,453 a 0,873 \\
\hline
\end{tabular}

\section{RESULTADOS}

\section{Fase 1}

\section{Tradução e adaptação cultural}

Na etapa da tradução, quando houve alguma divergência, optou-se por utilizar a versão do especialista por ser mais adequada à terminologia usada na prática médica. A etapa da retrotradução não apresentou discrepâncias relevantes, obtendo-se a versão traduzida do Nips para o pré-teste. Na etapa de adaptação semântica e cultural, foram indicadas sugestões e/ ou observações de alterações de dois itens do instrumento de pesquisa: "eu não faço uso efetivo do meu tempo profissional com aconselhamento nutricional" para "eu não farei uso efetivo do meu tempo profissional com aconselhamento nutricional", justificado pelo fato de eles ainda não realizarem atendimento de pacientes, e "a maioria dos pacientes tenta mudar seu estilo de vida se eu os aconselho a fazê-lo" para "a maioria dos pacientes mudaria o seu estilo de vida caso fossem aconselhados por profissionais de saúde".

Além disso, observou-se que não houve concentração de respostas nas extremidades nas questões que originalmente eram dicotômicas (sim ou não) e que foram alteradas para uma escala de resposta do tipo Likert de cinco pontos (onde 1 significa "discordo totalmente" e 5 significa "concordo totalmente"). Assim, no pré-teste foi possível assegurar que o questionário estava compreensível e passível de ser respondido. Encerrou-se a primeira fase da pesquisa com a conclusão da versão final, que foi o instrumento de coleta de dados da segunda fase.

\section{Fase 2}

\section{Verificação da validade do Nips}

Inicialmente, utilizou-se o índice Kaiser-Meyer-Olkin (KMO) para verificar a fatoriabilidade da matriz de correlação entre os itens da escala, obtendo-se o valor de 0,86 para a amostra estudada. A análise fatorial exploratória, utilizando o método dos componentes principais e rotação Varimax, indicou a retirada de oito questões devido à baixa proporção de variância explicada, cargas fatoriais baixas ou cargas fatoriais semelhantes em vários fatores. Houve uma redistribuição das questões restantes do questionário em dez fatores com autovalores maiores do que 1,00, explicando 58,9\% da variância total (Tabela 2).

O coeficiente alfa de Cronbach foi utilizado para avaliar a confiabilidade da consistência interna das questões que compõem cada um dos dez fatores latentes gerados pela análise fatorial. Sete dos dez fatores obtidos apresentaram medidas de alfa de Cronbach superiores a 0,60, consideradas satisfatórias; dois fatores apresentaram medidas acima de 0,50, consideradas suficientes; e somente um fator apresentou valores abaixo de 0,50. Estes resultados indicam confiabilidade da consistência interna de moderada a satisfatória. Optou-se por manter no instrumento os fatores que apresentaram valores inferiores a 0,60 , pois se constatou que a retirada de qualquer questão não geraria um ganho expressivo de confiabilidade do instrumento.

Ao se compararem as duas versões do questionário, observou-se que houve aumento de fatores e redistribuição dos 
TABELA 2

Cargas fatoriais, comunalidade, MSA, média, alfa de Cronbach, variância de cada escala e variância explicada pelo modelo Questões/fatores

1. Cuidado preventivo de saúde é chato. (INV)

2. Aconselhamento nutricional deve ser parte do atendimento de rotina de qualquer médico, independentemente da especialidade.

3. Avaliação nutricional e aconselhamento deveriam ser incluídos em qualquer consulta, assim como diagnóstico e tratamento.

4. Eu farei uso efetivo do meu tempo profissional com aconselhamento nutricional. Cargas fatoriais

5. O médico isoladamente tem pouco impacto na capacidade do paciente para perder peso.

F7

F1 F2

6. Eu tenho a obrigação de melhorar a saúde dos meus pacientes, inclusive discutindo 6. Eu tenho a obrigaça
nutrição com eles.

7. Todos os médicos, independentemente da especialidade, devem aconselhar pacientes de alto risco sobre mudanças alimentares.

8. Não vale a pena gastar tempo com aconselhamento nutricional se os pacientes têm padrão precário de nutrição. (INV)

10. É importante que eu fale da importância da dieta toda vez que eu cuidar de um paciente.

13. É importante que eu avalie o peso de cada paciente de acordo com as diretrizes de identificação, avaliação e tratamento do sobrepeso e obesidade em adultos, da OMS.

14. É importante que eu avalie a ingestão de vitaminas, minerais e suplementos dietéticos de cada paciente.

15. É importante que eu aconselhe pacientes em relação ao uso de suplementos e que chame a atenção quando são contraindicados.

16. É importante que eu encaminhe pacientes com problemas relacionados à dieta a nutricionistas ou outros profissionais qualificados

17. É importante que eu, sempre que possível, recomende mudanças na alimentação antes de iniciar tratamentos farmacológicos.

18. É importante que eu avalie a ingestão de gordura, fibra, fruta e vegetais de cada paciente, como estratégia preventiva.

19. É importante que eu solicite que os pacientes tragam um inventário alimentar ou que eu faça uma nova avaliação alimentar toda vez que o paciente vier para uma consulta de rotina. 20. É importante que eu encoraje pacientes a perguntar sobre suas dúvidas relacionadas à sua alimentação e encaminhá-los para assistência adicional quando possível.

21. É importante que eu avalie a ingestão alcoólica dos pacientes como parte de sua avaliação nutricional global.

22. É importante que eu avalie a intenção de mudança de cada paciente antes de iniciar intervenção nutricional.

23. É importante que eu avalie a ingestão de sódio, potássio e cálcio na dieta, especialmente em pacientes com risco de hipertensão, osteoporose ou derrame.

24. É importante que eu encaminhe pacientes diabéticos para aconselhamento nutricional específico.

25. É importante que eu defenda o equilíbrio entre nutrição e exercícios para promover controle de peso

28. É importante que eu avalie a capacidade do meu paciente de entender a tabela nutricional presente na etiqueta dos alimentos.

29. A motivação do paciente é essencial para obter mudanças alimentares.

0,78

0,78

0,79

0,64

0,76

30. A maioria dos pacientes obesos quer perder peso, mas eles se sentem frustrados e confusos em relação a como fazê-lo.

31. Pacientes precisam de alternativas saborosas para conseguir mudar seu padrão alimentar.

32. Uma mudança visando a um estilo de vida mais saudável é importante em qualquer fase da vida.

34. Pacientes precisam de instruções específicas de como mudar seus hábitos alimentares. 35. Aconselhamento específico sobre como fazer mudanças na alimentação pode ajudar alguns pacientes a melhorar seus hábitos alimentares.

36. Pacientes precisam de um aconselhamento contínuo após as instruções iniciais para que eles mantenham as mudanças de comportamento consistentes com uma dieta mais saudável. 37. Pacientes não são motivados a mudar a não ser que estejam doentes.

38. Pacientes só mudarão seus hábitos alimentares se estiverem enfrentando um grave problema de saúde, como, por exemplo um infarto.

39. Pacientes raramente mudarão seu comportamento se não têm sintomas de alguma doença.

40. A maioria dos pacientes mudaria seu estilo de vida caso fosse aconselhada por profissionais da saúde.

43. Após receber aconselhamento nutricional, pacientes com maus hábitos alimentares farão uma mudança significativa no seu comportamento alimentar.

44. Os meus esforços na educação dos meus pacientes serão efetivos em aumentar a adesão dos mesmos às recomendações nutricionais.

45. Após receber aconselhamento nutricional, pacientes com maus hábitos alimentares farão alguma mudança nos seus hábitos alimentares.

Alfa de Cronbach

\% variância explicada

Autovalor

0,64 0,76

$\begin{array}{lll}0,667 & 0,749 \quad 4,0 & (0,9)\end{array}$

$0,574 \quad 0,739 \quad 3,5(1,1)$

0,61

0,68

$\begin{array}{lll}0,555 & 0,941 \quad 4,5 & (0,6)\end{array}$ $0,653 \quad 0,876 \quad 4,1(0,8)$

$0,567 \quad 0,902 \quad 4,4(0,6)$

$\begin{array}{lll}0,669 & 0,836 & 4,0 \\ 0,6,8)\end{array}$

$0,634 \quad 0,693 \quad 2,7(1,1)$

$\begin{array}{lll}0,789 & 0,601 \quad 2,5(1,1)\end{array}$

$0,672 \quad 0,643 \quad 2,9(1,1)$

$0,482 \quad 0,740 \quad 3,3(0,9)$

$0,575 \quad 0,714 \quad 3,0(0,9)$

$0,503 \quad 0,867 \quad 3,8(0,7)$

$0,642 \quad 0,724 \quad 3,6(0,8)$ 0,77

$\begin{array}{cccccccccc}0,78 & 0,78 & 0,72 & 0,66 & 0,78 & 0,71 & 0,62 & 0,51 & 0,55 & 0,24 \\ 10,41 & 7,66 & 6,60 & 6,12 & 5,96 & 5,16 & 4,74 & 4,28 & 4,18 & 3,76 \\ 3,86 & 2,84 & 2,44 & 2,27 & 2,21 & 1,91 & 1,76 & 1,58 & 1,55 & 1,40\end{array}$

\section{Total variância explicada}

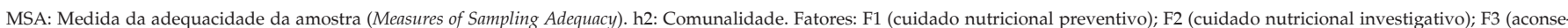

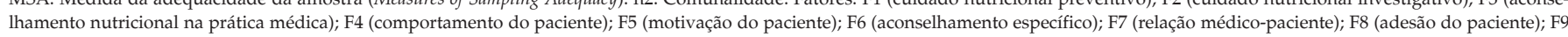

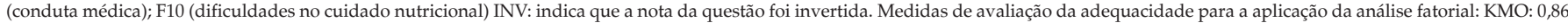
e teste de esfericidade de Bartlett: $\mathrm{p}<0,0001$. 
itens na versão traduzida. Deste modo, houve a necessidade de modificar os nomes dos fatores de modo a explicar melhor os subconceitos dentro de uma mesma dimensão.

\section{Versão final do Nips validado para a língua portuguesa}

Assim, concluído o processo de análise fatorial, foi definida a estrutura final da escala, que passou a ter 37 itens em formato Likert de 1 (discordo totalmente) a 5 (concordo totalmente) (Quadro 1). Os itens se organizaram em dez fatores, nomeados conforme demonstrado a seguir:

(A) Cuidado nutricional preventivo: composto por sete itens que avaliam a percepção do estudante de Medicina sobre a importância da atuação do médico na promoção de mudanças de hábitos alimentares no cuidado preventivo. Este fator corresponde a 10,4\% da porcentagem de variância explicada, com alfa de Cronbach 0,78 ;

(B) Cuidado nutricional investigativo: constituído por seis itens que avaliam a percepção do estudante de Medicina sobre a necessidade de o médico fazer uma avaliação nutricional do paciente no cuidado de rotina. Este fator corresponde a 7,6\% da porcentagem de variância explicada, com alfa de Cronbach 0,78 ;

(C) Aconselhamento nutricional na prática clínica: formado por quatro itens, destina-se a identificar a intenção de destinar um tempo da consulta ao aconselhamento nutricional do paciente. Este fator corresponde a $6,6 \%$ da porcentagem de variância explicada, com alfa de Cronbach 0,72 ;

(D) Comportamento do paciente: composto por quatro itens que avaliam a percepção dos estudantes sobre o comportamento dos pacientes após aconselhamento nutricional. Este fator corresponde a 6,1\% da porcentagem de variância explicada, com alfa de Cronbach 0,66;

(E) Motivação do paciente: constituído por três itens que avaliam a percepção dos fatores relacionados com a motivação dos pacientes para mudar seus hábitos alimentares e explica 5,9\% da variância, com alfa de Cronbach 0,78,

(F) Aconselhamento específico: composto por três itens que avaliam a percepção dos estudantes sobre a importância do aconselhamento nutricional específico na mudança de hábitos do paciente e explica 5,1\% da variância, com alfa de Cronbach 0,71;

(G) Relação médico-paciente: constituído por quatro itens que avaliam a percepção da importância do relacionamento médico-paciente no cuidado nutricional e explica $4,7 \%$ da variância, com alfa de Cronbach 0,62;

(H) Adesão do paciente, formado por dois itens que se destinam a identificar a necessidade de o paciente entender como deve proceder para não se frustrar com a dieta e a im- portância de alternativas saborosas para a adesão à dieta e explica $4,2 \%$ da variância, com alfa de Cronbach 0,51 ;

(I) Conduta médica, composto por dois itens, que avaliam a percepção dos estudantes sobre a obrigação do médico de fazer o aconselhamento nutricional a fim de melhorar a saúde do paciente e explica $4,1 \%$ da variância, com alfa de Cronbach 0,55 ;

(J) Dificuldades no cuidado nutricional, constituído por dois itens que se referem à percepção de que cuidados preventivos são chatos e que o médico tem pouca capacidade de modificar os hábitos dos pacientes e explica 3,7\% da variância, com alfa de Cronbach 0,24.

O coeficiente alfa de Cronbach foi utilizado para avaliar a confiabilidade da consistência interna das questões que compõem cada um dos dez fatores latentes gerados pela análise fatorial. Ele mostra que sete dos dez fatores obtidos apresentam medidas de alfa de Cronbach superiores a 0,60, indicando confiabilidade satisfatória de consistência interna.

\section{DISCUSSÃO}

Avaliar a atitude e o comportamento de estudantes de Medicina a respeito do poder do médico e do cuidado na atenção ao paciente vem sendo alvo de estudos ${ }^{2,11,12}$. Atitude é definida como a predisposição para responder de certa maneira frente a um objeto ou pessoa. Por se tratar de um construto subjetivo, necessita ser medido por metodologias específicas. $\mathrm{Na}$ medicina, uma ferramenta frequentemente utilizada para esta finalidade são as escalas de autorrespostas, que permitem medir a intensidade de sentimentos referentes a um objeto, com a classificação de afirmativas que variam de favorável a desfavorável, em graus variados (escala de Likert) ${ }^{20}$.

Embora as escalas de medidas de atitude comportem críticas, como não é possível ver uma atitude, essas escalas permitem inferir sua magnitude. A validade de escalas constitui um parâmetro de medida tipicamente discutida no campo da educação médica ${ }^{21}$. O uso de técnicas psicométricas na educação decorreu da necessidade de determinar o grau de aptidão dos indivíduos avaliados por meio de instrumentos válidos e confiáveis. Assim, a psicometria procura expressar numericamente os fenômenos psicológicos em vez de empregar uma descrição verbal ${ }^{18}$. A validação interna atribui qualidade ao instrumento de medição. Uma boa mensuração, segundo Hubley e Zumbo ${ }^{22}$, deve ter duas características essenciais: confiabilidade e validade. A confiabilidade é sinônimo de consistência, estabilidade e previsibilidade. Já a validade tem um sentido de acuracidade, autenticidade e veracidade.

Segundo Pasquali ${ }^{18}$, a confiabilidade ou fidedignidade se relaciona com a capacidade de um instrumento medir com precisão o que se propõe medir. Entre os métodos para a 


\section{QuADro1}

\section{Versão final do NIPS validado no Brasil}

Cuidado nutricional preventivo

1. É importante que eu encaminhe pacientes com problemas relacionados à dieta a nutricionistas ou outro profissional qualificado.

$\square \quad \square \quad \square \quad \square$

2. É importante que eu encoraje pacientes a perguntar sobre suas dúvidas relacionadas à sua alimentação e encaminhá-los para assistência adicional quando possível.

3. É importante que eu encaminhe pacientes diabéticos para aconselhamento nutricional específico.

4. É importante que eu defenda o equilíbrio entre nutrição e exercícios para promover controle de peso.

5. É importante que eu auxilie pacientes pediátricos a estabelecer hábitos alimentares saudáveis precocemente para prevenir o risco de doenças crônicas.

6. A motivação do paciente é essencial para obter mudanças alimentares.

7. Uma mudança visando a um estilo de vida mais saudável é importante em qualquer fase da vida.

\section{Cuidado nutricional investigativo}

8. É importante que eu avalie o peso de cada paciente de acordo com as diretrizes de identificação, avaliação e tratamento do sobrepeso e obesidade em adultos, da OMS.

9. É importante que eu avalie a ingestão de vitaminas, minerais e suplementos dietéticos de cada paciente.

10. É importante que eu aconselhe pacientes em relação ao uso de suplementos e que chame a atenção quando são contraindicados.

11. É importante que eu avalie a ingestão de gordura, fibra, fruta e vegetais de cada paciente, como estratégia preventiva.

12. É importante que eu solicite que os pacientes tragam um inventário alimentar ou que eu faça uma nova avaliação alimentar toda vez que o paciente vier para uma consulta de rotina.

13. É importante que eu avalie a ingestão de sódio, potássio e cálcio na dieta, especialmente em pacientes com risco de hipertensão, osteoporose ou derrame.

\section{Aconselhamento nutricional na prática clínica}

14. Aconselhamento nutricional deve ser parte do atendimento de rotina de qualquer médico, independentemente da especialidade.

15. Avaliação nutricional e aconselhamento deveriam ser incluídos em qualquer consulta, assim como diagnóstico e tratamento.

16. Eu farei uso efetivo do meu tempo profissional com aconselhamento nutricional.

17. É importante que eu fale da importância da dieta toda vez que eu cuidar de um paciente.

\section{Comportamento do paciente}

18. A maioria dos pacientes mudaria seu estilo de vida caso fosse aconselhada por profissionais da saúde.

19. Após receber aconselhamento nutricional, pacientes com maus hábitos alimentares farão uma mudança significativa no seu comportamento alimentar.

20. Os meus esforços na educação dos meus pacientes serão efetivos em aumentar a adesão dos mesmos às recomendações nutricionais. $\quad \square \quad \square \quad \square \quad \square \quad \square$ 21. Após receber aconselhamento nutricional, pacientes com maus hábitos alimentares farão alguma mudança nos seus hábitos alimentares.

\section{Motivação do paciente}

22. Pacientes não são motivados a mudar a não ser que estejam doentes.

23. Pacientes só mudarão seus hábitos alimentares se estiverem enfrentando um grave problema de saúde, como, por exemplo, um infarto.

24. Pacientes raramente mudarão seu comportamento se não têm sintomas de alguma doença.

\section{Aconselhamento específico}

25. Pacientes precisam de instruções específicas de como mudar seus hábitos alimentares.

26. Aconselhamento específico sobre como fazer mudanças na alimentação pode ajudar alguns pacientes a melhorar seus hábitos alimentares.

27. Pacientes precisam de um aconselhamento contínuo após as instruções iniciais para que eles mantenham as mudanças de comportamento consistentes com uma dieta mais saudável.

\section{Relação médico-paciente}

28. Não vale a pena gastar tempo com aconselhamento nutricional se os pacientes têm padrão precário de nutrição.

29. É importante que eu, sempre que possível, recomende mudanças na alimentação antes de iniciar tratamentos farmacológicos.

30. É importante que eu avalie a ingestão alcoólica dos pacientes como parte de sua avaliação nutricional global.

31. É importante que eu avalie a intenção de mudança de cada paciente antes de iniciar intervenção nutricional.

\section{Adesão do paciente}

32. A maioria dos pacientes obesos quer perder peso, mas eles se sentem frustrados e confusos em relação a como fazê-lo.

33. Pacientes precisam de alternativas saborosas para conseguir mudar seu padrão alimentar.

\section{Conduta médica}

34. Eu tenho a obrigação de melhorar a saúde dos meus pacientes, inclusive discutindo nutrição com eles.

35. Todos os médicos, independentemente da especialidade, devem aconselhar pacientes de alto risco sobre mudanças alimentares.

Dificuldades no cuidado nutricional

36. Cuidado preventivo de saúde é chato.

37. O médico isoladamente tem pouco impacto na capacidade do paciente para perder peso.

$\begin{array}{lllll}1 & 2 & 3 & 4 & 5\end{array}$

$\square \quad \square \quad \square \quad \square$

$\square \quad \square \quad \square \quad \square$

$\square \quad \square \quad \square \quad \square$

$\begin{array}{lllll}1 & 2 & 3 & 4 & 5\end{array}$

$\square \quad \square \quad \square \quad \square$

$\square \quad \square \quad \square \quad \square$

$\square \quad \square \quad \square \quad \square$ 
estimação da confiabilidade, pode-se citar o método da consistência interna. Entre os métodos utilizados para medir a consistência interna, o cálculo do alfa de Cronbach é o mais empregado nos trabalhos acadêmicos atuais.

Neste estudo, a análise fatorial conduzida demonstrou que existe um grau satisfatório de interdependência entre os itens, uma vez que as medidas de comunalidade e adequacidade apresentaram valores superiores a 0,50. Observou-se também que os itens se distribuíram em dez fatores, cujos autovalores foram superiores a 1, sendo que a amplitude dos domínios abordados nesse instrumento explica 58,93\% das atitudes dos alunos em relação ao cuidado nutricional na assistência ao paciente. Ao compararmos a versão traduzida do Nips com a versão original, constatamos que houve grande redistribuição dos itens e aumento dos fatores. Entretanto, observou-se que em dois fatores - comportamento do paciente e motivação do paciente - a versão traduzida permaneceu com o mesmo agrupamento de itens apresentado na versão original. As questões que eram dicotômicas na versão original se distribuíram em três fatores, mostrando haver maior distinção entre os domínios avaliados por elas, o que foi possível observar com uma escala mais abrangente (como é o caso da escala de Likert). As questões do domínio original "relacionamento médico-paciente" dividiram-se em dois domínios distintos. As questões do domínio "nutrição no atendimento de rotina" dividiram-se em três fatores distintos, sendo que somente uma das questões deste fator foi realocada junto com questões de outros fatores. Assim, no geral, observou-se que, apesar da redistribuição das questões, estas se mantiveram, na maioria dos casos, agrupadas de forma semelhante ao agrupamento da versão original, entretanto com subdivisão dos fatores.

É possível que as diferenças encontradas entre as duas versões do instrumento estejam relacionadas com as características inerentes à amostra utilizada: estudantes de Medicina, período do curso, particularidades do curso de Medicina pesquisado, além dos aspectos da cultura brasileira ${ }^{23}$. Estas discrepâncias observadas podem, também, estar relacionadas com alguns aspectos da análise fatorial do artigo original. Notou-se que várias questões da versão original apresentaram medidas de comunalidade e cargas fatoriais baixas. Além disso, o valor da porcentagem de variância explicada foi muito baixo (36\%). McGaghie et al. ${ }^{13}$ ressaltam, como limitação do estudo, as incongruências frequentemente observadas no desenvolvimento de escalas entre a análise estatística e a escolha de itens para a composição da escala. Segundo esses autores, o equilíbrio entre o julgamento intuitivo e a ciência psicométrica faz-se necessário de modo a capturar a "arte do desenvolvimento de escalas de atitudes".
Assim, o Nips validado mostrou ser um instrumento com qualidades psicométricas apropriadas. Os valores encontrados para o alfa de Cronbach mostram níveis de moderados a satisfatórios de confiabilidade, uma vez que em nove dos dez fatores os valores foram maiores que $0,50^{18}$.

Acrescenta-se ainda que, a escala Nips é facilmente compreendida pelos estudantes, bem como proporciona facilidade de aplicação, tanto individual como coletiva. As dimensões do Nips permitem obter uma ampla gama de informações relativas às percepções dos estudantes sobre as questões que permeiam o aconselhamento nutricional do paciente.

O presente estudo foi limitado a estudantes de Medicina e, portanto, seu uso não pode ser extrapolado para outras populações. No entanto, ressalta-se a intenção de se prosseguir com estudos de validação desta escala, incluindo residentes e outros estudantes de áreas ligadas à saúde.

\section{CONCLUSÃO}

A versão final brasileira do Nips atendeu aos critérios de equivalência semântica, idiomática, cultural e conceitual. Conclui-se que o Nips validado pode ser aplicado em nosso meio, pois possibilita identificar as atitudes dos estudantes de Medicina em relação à assistência nutricional na prática clínica. Espera-se que o instrumento Nips validado possa contribuir para o diagnóstico das atitudes dos alunos de Medicina em relação à nutrição e posterior desenvolvimento de políticas de gestão acadêmica, bem como para o aprimoramento das práticas docentes.

Assim, intervenções para capacitar os futuros médicos quanto ao cuidado nutricional na prática clínica poderão ajudar a conscientizar os pacientes da importância de hábitos alimentares saudáveis e, com isso, contribuir para sanar os graves problemas de sobrepeso e obesidade e suas repercussões na saúde. Mais estudos devem ser realizados com o intuito de estender a utilização do instrumento a residentes, médicos e outros profissionais da área de saúde.

\section{REFERÊNCIAS}

1. Organização Pan-Americana de Saúde. Modelo de Perfil Nutricional da Organização Pan-Americana da Saúde. Washington; 2016.

2. Hseiki RA, Osman MH, El-Jarrah RT, Hamadeh GN, Lakkis NA. Knowledge, attitude and practice of Lebanese primary care physicians in nutrition counseling: a self-reported survey. Prim Health Care Res Dev 2017; 18(06):629-634.

3. Oliveira JED, Marchini JS. Nutrologia: especialidade médica. Rev Assoc Med Bras 2008;54(6):471-486.

4. Sacks FM, Svetkey LP, Vollmer WM, Appel LJ, Bray GA, Harsha D, et al. Effects on blood pressure of reduced dieta- 
ry sodium and the Dietary Approaches to Stop Hypertension (DASH) diet. DASH-Sodium Collaborative Research Group. N Engl J Med 2001; 344(1):3-10.

5. Look AHEAD Research Group, Wing RR. Long term effects of a lifestyle intervention on weight and cardiovascular risk factors in individuals with type 2 diabetes: four-year results of the Look AHEAD trial. Arch Intern Med 2010;170(17):1566-75.

6. Polak R, Pojednic RM, Phillips EM. Lifestyle Medicine Education. Am J Lifestyle Med 2015;9(5):361-367.

7. Lampert JB. Dois séculos de escolas médicas no Brasil e a avaliação do ensino médico no Panorama atual e perspectivas. Gaz Méd Bahia 2008;78(1):31-37.

8. Kopelman P, Lennard-Jones J. Nutrition and patients: a doctor's responsibility. Clin Med (Lond) 2002;2(5):391-394.

9. Spencer EH, Frank E, Elon LK, Hertzberg VS, Serdula MK, Galuska DA. Predictors of nutrition counseling behaviors and attitudes in US medical students. Am J Clin Nutr 2006;84(3):655-662.

10. Conroy MB, Delichatsios HK, Hafler JP, Rigotti NA. Impact of a preventive medicine and nutrition curriculum for medical students. Am J Prev Med 2004;27(1):77-80.

11. Adams KM, Kohlmeier M, Zeisel SH. Nutrition Education in U.S. Medical Schools: Latest Update of a National Survey. Acad Med 2010;85(9):1537-42.

12. Womersley K, Ripullone K. Medical schools should be prioritising nutrition and lifestyle education. BMJ 2017;359:j4861.

13. McGaghie WC, Van Horn L, Fitzgibbon M, Telser A, Thompson JA, Kushner RF, et al. Development of a measure of attitude toward nutrition in patient care. Am J Prev Med 2001;20(1):15-20.

14. Walsh CO, Ziniel SI, Delichatsios HK, Ludwig DS. Nutrition attitudes and knowledge in medical students after completion of an integrated nutrition curriculum compared to a dedicated nutrition curriculum: a quasi-experimental study. BMC Med Educ 2011;11:58.

15. Crowley J, Ball L, Han DY, McGill AT, Arroll B, Leveritt M, et al. Doctors' attitudes and confidence towards providing nutrition care in practice: Comparison of New Zealand medical students, general practice registrars and general practitioners. J Prim Health Care 2015;7(3):244-250.

16. Anderson LW. Attitudes and their Measurement. In: Walberg HJ.; Haertel G. D. (Ed.). The International Encyclopedia of Educational Evaluation. Oxford: Pergamon, 1990. P. 368-374.
17. Beaton D, Bombardier C, Guillemin F, Ferraz M B. Recommendations for the cross-cultural adaptation of health status measures. American Academy of Orthopedic Surgeons Institute for Work \& Health. 2002. [acesso 14 abr 2019]. Disponível em: http:/ / www.dash.iwh.on.ca/assets/images / pdfs/xculture2002.pdf.

18. Pasquali L. Psicometria: teoria dos testes na Psicologia e na Educação. 4. ed. Petrópolis: Vozes; 2011.

19. Hair JF, Black WC, Babin BJ, Anderson RE, Tatham RL. Análise Multivariada de Dados. 5. ed. Porto Alegre: Brookman, 2005.

20. Ribeiro MMF, Amaral CFS. Medicina centrada no paciente e ensino médico: a importância do cuidado e o poder médico. Rev Bras Educ Med. 2008; 32(1)90-97.

21. Kogan JR, Shea JA. Course evaluation in medical education. TeachTeach Educ. 2007;23(3)251-64.

22. Hubley A, Zumbo, BD. A dialectic on validity: where we have been and where we are going. Journal of General Psychology, 1996;23(3):207-215.

23. Wild D, Eremenco S, Mear I, Martin M, Houchin C, Gawlicki $\mathrm{M}$, et al. Multinational trials recommendations on the translations required, approaches to using the same language in different countries, and the approaches to support pooling the data: the ISPOR Patient Reported Outcomes Translation and Linguistic Validation Good Research Practices Task Force report. Value Health. 2009;12(4)430-40.

\section{CONTRIBUIÇÃO DOS AUTORES}

Juliana Peres Sleumer participou do delineamento da pesquisa, da coleta de dados, da análise dos dados, da redação e da revisão do artigo. Ângelo Ponte de Freitas Campos participou da coleta de dados, da redação e da revisão do artigo. Felipe Anastácio da Silva Machado participou da coleta de dados, da redação e da revisão do artigo. Eliane Perlatto Moura participou do delineamento da pesquisa, da coleta de dados, da redação e da revisão do artigo.

\section{CONFLITO DE INTERESSES}

Os autores declaram não haver conflito de interesses neste estudo.

\section{ENDEREÇO PARA CORRESPONDÊNCIA}

Eliane Perlatto Moura

Rua Tomé de Souza 1322 apto 1201

Funcionários - Belo Horizonte - MG - CEP: 30140130

E-mail: elianeperlatto@gmail.com 